\title{
CDHII inhibits proliferation and invasion in head and neck cancer
}

\author{
Songlin Piao ${ }^{1,2}$, Ronald C. Inglehart ${ }^{1}$, Christina Springstead Scanlon, Nickole Russo', Rajat Banerjee, \\ Nisha J. D'Silva ${ }^{1,3}$ \\ ${ }^{1}$ Department of Periodontics and Oral Medicine, School of Dentistry, University of Michigan, Ann Arbor, MI, USA; ${ }^{2}$ Department of Oral \\ and Maxillofacial Surgery, The First Affiliated Hospital of Harbin Medical University, Harbin, Heilongjiang, China; ${ }^{3}$ Department of \\ Pathology, University of Michigan Medical School, University of Michigan, Ann Arbor, MI, USA
}

\begin{abstract}
BACKGROUND: In this study, we use a bioinformaticsbased strategy to nominate a tumor suppressor gene cadherin-I I (CDHII) and investigate its role in growth and invasion in head and neck squamous cell carcinoma (HNSCC).

METHODS: Using the Oncomine ${ }^{T M}$ database to compare HNSCC and normal specimens, CDHII was nominated as having a role in HNSCC. CDHI I expression in HNSCC was evaluated by immunohistochemistry on a tissue microarray (TMA) and immunoblotting and immunofluorescence of cell lines. The functional impact of CDH I on proliferation and invasion was evaluated after siRNAmediated knockdown.

RESULTS: In silico analysis suggested that CDHII is overexpressed in HNSCC compared to normal specimens. HNSCC TMA exhibited a small but significant increase in intensity and proportion of CDHII. By immunoblot analysis, CDHII was higher in 4/7 HNSCC cell lines compared to normal keratinocytes; CDHI I was highly upregulated in UM-SCC-47 and UM-SCC-74A and detectable in UM-SCC-I4A and UM-SCC-29 cell lines. Downregulation of CDHII in both UM-SCC-29 and UM-SCC-47 using two different siRNAs enhanced proliferation and invasion.

CONCLUSION: CDHII inhibits cell proliferation and invasion of HNSCC. This suggests that CDHI I functions as a tumor suppressor gene in head and neck cancer. Our findings emphasize the importance of verifying in silico findings with functional studies.
\end{abstract}

J Oral Pathol Med (2017) 46: 89-97

Keywords: CDHI I; meta-analysis; squamous cell carcinoma

Correspondence: Nisha J. D'Silva, Department of Periodontics and Oral Medicine, School of Dentistry, University of Michigan, 1011 North University Ave, Room 5217, Ann Arbor, MI 48109-1078, USA. Tel: (734) 764 1543, Fax: (734) 764 2469, E-mail: njdsilva@umich.edu

Accepted for publication May 25, 2016

\section{Introduction}

In silico analysis of existing datasets has become an important approach in identifying tumor-specific networks and nominating molecular targets in head and neck squamous cell carcinoma (HNSCC) (1). Translational researchers use in silico analyses linked to clinical data to complement laboratory research. Meta-analyses of existing microarray data demonstrate correlations of markers across datasets within a cancer type and across clinical stages. As the importance of in silico analyses increases in translational research studies, multiple platforms have been assembled for bioinformatics analysis. The present study used Oncomine ${ }^{\mathrm{TM}}$, which is a platform for meta-analysis of microarray datasets each of which includes multiple normal and tumor samples (2). Using this approach, we identified that Cadherin11 (CDH11), also known as osteoblasts-cadherin, is differentially regulated in HNSCC and normal tissue.

CDH11, one of the type II classical cadherins, was first identified in osteoblasts where it is an integral membrane protein involved in cell-cell adhesion, potentially playing a role in development and maintenance of bone (3). CDH11 promotes invasion and metastasis in prostate and breast cancers, consistent with an oncogenic role $(4,5)$. In breast cancer, $\mathrm{CDH} 11$ promotes metastases to the bone, due to the high binding affinity of cancer cells for the strongly CDH11expressing osteoblasts. (4). In contrast, in esophageal, colorectal and gastric cancers, CDH11 is silenced by methylation and has a tumor suppressor role $(6,7)$. In esophageal and nasopharyngeal cancer cells, $\mathrm{CDH} 11$ inhibits invasion and migration (7). Due to the variability in CDH11 function, it is important to consider the cellular context. In the present study, using an in silico approach, we nominated CDH11 as having a role in HNSCC and investigated the expression and function of CDH11 in HNSCC.

\section{Materials and methods}

In silico studies

The Oncomine ${ }^{\mathrm{TM}}$ database (Compendia Bioscience, Ann Arbor, MI, USA) was used for in silico studies. Eighteen 
head and neck cancer datasets were identified using the search parameters 'Cancer Type: Head and Neck Cancer' and 'Analysis Type: Cancer vs. Normal Analysis'. Datasets from studies of adenoid cystic carcinoma, thyroid carcinomas and nasopharyngeal carcinomas were excluded. The following studies were retained: Cromer Head- Neck (8), Estilo Head-Neck (9), Ginos Head-Neck (10), Kuriakose Head-Neck (11), Peng Head-Neck and Peng Head-Neck 2 (12), Pyeon Multi-cancer (Tongue, Oropharyngeal, Floor of Mouth and Oral Cavity) (13), Schlingemann Head-Neck (14), Talbot Lung (included tongue) (15), Toruner HeadNeck (16), The Cancer Genome Atlas (TCGA) Head-Neck, Ye Head-Neck (17). A summary of CDH11 overexpression in multiple cancer types was generated. In addition, correlation between CDH11 and epithelial to mesenchymal transition (EMT) markers, E-Cadherin and Twist-1, was evaluated from datasets in Oncomine ${ }^{\mathrm{TM}}$.

GraphPad Prism ${ }^{\circledR}$ was used for statistical analyses. HNSCC datasets with mRNA expression data $(n=15)$ were identified in Oncomine ${ }^{\mathrm{TM}}$. Meta-analyses were performed as described (18). Expression of CDH11 was compared between normal and HNSCC samples by Student's $t$-test in each HNSCC dataset, and a $P$-value of $<0.05$ was determined to be statistically significant. Each statistically significant study was given an arbitrary value of ' 1 ' and non-significant studies were assigned a value of ' 0 '. A one-sample $t$-test was performed for each set of values, and a one-sided $P$-value of $<0.05$ was considered statistically significant.

\section{Immunohistochemistry}

Immunostaining on formalin-fixed, paraffin-embedded tissue and scoring of staining intensity was performed as described (18) on a HNSCC tissue microarray (TMA) (US Biomax. Rockville, MD, USA). The CDH11 affinity purified rabbit polyclonal antibody $(2.7 \mu \mathrm{g} / \mathrm{ml})$ used for immunohistochemical studies was from R\&D Systems. Rabbit IgG (Dako, Carpinteria, CA, USA) was used as a negative control at the same concentration as the primary antibody. For analysis of TMA data, interpretation and scoring were performed by a board-certified pathologist as described (18). The clinico-pathological parameters of the HNSCC cases and CDH11 intensity and proportion scores are included in Table S1.

\section{Cell culture}

UM-SCC cell lines were provided by Thomas Carey (University of Michigan). Cells were genotyped to confirm identity and cultured as described (18). All UM-SCC cell lines were maintained in Dulbecco's modified Eagle's medium (Gibco, Life Technologies, Grand Island, NY, USA) supplemented with $10 \%$ FBS and $1 \%$ penicillin/ streptomycin. Immortalized keratinocytes, HOK16B, obtained from Dr. NH Park (UCLA), were cultured as described.

\section{Transfection}

To downregulate CDH11, HNSCC cells were transfected with siRNA (Dharmacon, Lafayette, CO). The sequences for each siRNA were: Non-Target $5^{\prime}$-GUGAUUUCAUAGCGAGUUU-3', siCDH11-7 5'-GGAAAUAGCGCCAAGUU

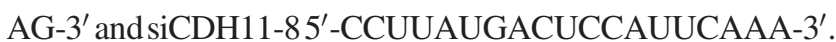
UM-SCC-47 and UM-SCC-29 were seeded in a 6-well plate and transfected with siRNA using RNAiMAX (Invitrogen, Carlsbad, CA, USA), according to the manufacturer's instructions.

\section{Immunoblot}

Lysates for detecting CDH11 were prepared using Red Loading Buffer Pack (Cell Signaling, Danvers, MA, USA). Lysates for detecting the other proteins were prepared using $1 \%$ NP40 lysis buffer (18). Antibodies used were CDH11 (A16652; Cell Signaling Technologies, Danvers, MA, USA), Twist-1 (25465-1-AP; Proteintech, Chicago, IL, USA), E-Cadherin (610182; BD Biosciences, Franklin Lakes, NJ, USA), GAPDH (AB2302; Millipore, Billerica, MA, USA), and Actin (612656; BD Biosciences).

\section{Immunofluorescence staining}

Cells were stained with E-cadherin (610182; BD Biosciences) in $0.3 \%$ triton $\mathrm{X}-100$ overnight at $4^{\circ} \mathrm{C}$. Cells were washed and incubated with secondary antibody and imaged on a Nikon Eclipse Ti microscope.

In vitro proliferation and invasion assay

At $24-48 \mathrm{~h}$ post-transfection, cells were seeded at equal densities $\left(1 \times 10^{4}\right.$ for UM-SCC-29, $2 \times 10^{4}$ for UM-SCC47). Viable, non-viable, and total cells were quantified at 1 , 3 , and 5 days using the Countess assay system (Invitrogen) with trypan blue.

Cell invasion was assessed 24-72 h after transfection using the modified Boyden chamber assay with Transwell inserts coated with Matrigel (BD Biosciences), as described (18). Inserts, not coated with matrigel, were used as a control for migration.

\section{Statistical analysis}

$P$-values were calculated using the student's $t$-test, a $P$-value of less than 0.05 was accepted as significant. Correlation coefficients (Spearman correlation) denoted by $r$, together with a $P$-value, were computed to measure correlation between different genes.

\section{Results}

HNSCC datasets provide extensive data for in silico studies The Oncomine ${ }^{\mathrm{TM}}$ database contained 986 datasets and 89461 samples (accessed 04/24/2015). Of the available datasets, 36 were identified as 'Head and Neck Cancer' datasets. Figure 1 gives an overview of the content of these datasets. DNA or mRNA expression data are available for the datasets (Fig. 1A). While most datasets contain less than 75 samples, about $20 \%$ contain more than 151 samples (Fig. 1B). The data provided come from a range of sources, including cell panels, tissue panels and TCGA, which was compiled by the National Cancer Institute and National Human Genome Research Institute (Fig. 1C). The specimens used to generate the datasets include surgical specimens and samples collected by laser-capture microdissection, manual microdissection, and macrodissection (Fig. 1D). Several available search platforms for head and neck cancer dataset analysis are listed in Table 1. 
A

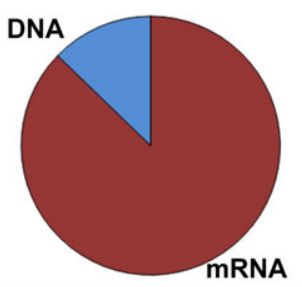

C

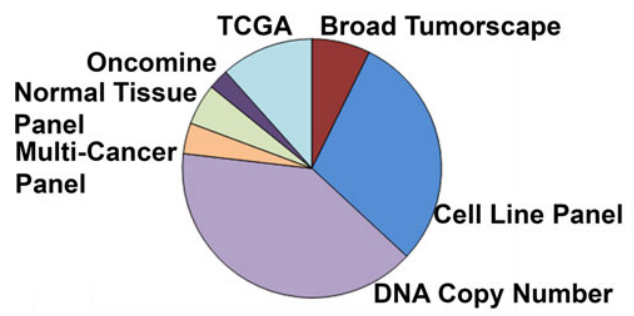

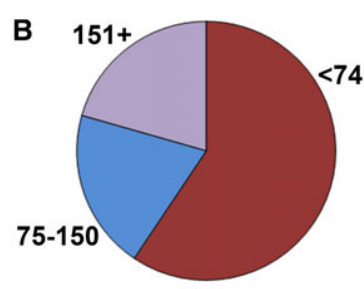

D

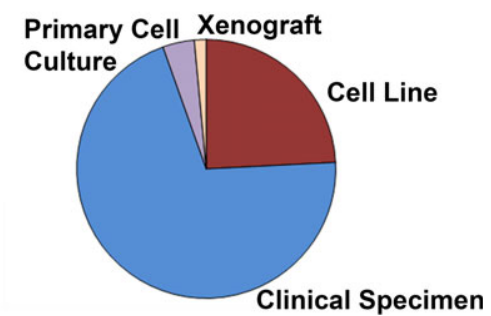

Figure 1 Details of Head-Neck Cancer datasets. (A) A larger number of datasets provide mRNA expression (87\%) levels than DNA copy-number analyses (13\%). (B) While most of the sample sets have $<75$ samples (59\%), $20 \%$ contain more than 151 samples. (C) Data are supplied by a range of sources including cell and tissue and data from sources including The Cancer Genome Atlas (TCGA), which is compiled by the National Cancer Institute and National Human Genome Research Institute. (D) Samples represent a variety of sources, including cell lines, surgical specimens, and both macro and micro dissections.

Table 1 Sample databases available for in silico studies in tumor/normal specimens

\begin{tabular}{|c|c|c|}
\hline Database & Access information & Description \\
\hline Cancer Gene Expression Database & $\begin{array}{l}\text { http://lifesciencedb.jp/cged/ } \\
\text { Freely searchable. }\end{array}$ & $\begin{array}{l}\text { A database of gene expression from studies performed in } \\
\text { Japanese institutions. }\end{array}$ \\
\hline $\begin{array}{l}\text { GENT - Gene Expression Across } \\
\text { Normal and Tumor tissue }\end{array}$ & $\begin{array}{l}\text { http://medical-genome.kribb.re.kr/GENT/ } \\
\text { Freely searchable. }\end{array}$ & $\begin{array}{l}\text { A database of comparative gene expression across normal } \\
\text { and cancer tissues. }\end{array}$ \\
\hline $\begin{array}{l}\text { KEGG Database (Kyoto Encyclopedia } \\
\text { of Genes and Genomes) }\end{array}$ & $\begin{array}{l}\text { http://www.genome.jp/kegg/kegg } 1 . h t m l \\
\text { Freely searchable. }\end{array}$ & $\begin{array}{l}\text { An integrated database of genomic and functional data, } \\
\text { links genes to higher level functions. }\end{array}$ \\
\hline Oncomine & $\begin{array}{l}\text { https://www.oncomine.org/ } \\
\text { Registration available to users from } \\
\text { educational, government and } \\
\text { non-profit institutions. }\end{array}$ & $\begin{array}{l}\text { A compilation of cancer microarray data, search focus } \\
\text { can be narrowed very finely. }\end{array}$ \\
\hline $\begin{array}{l}\text { RefSeq - The Reference } \\
\text { Sequence Collection }\end{array}$ & $\begin{array}{l}\text { http://www.ncbi.nlm.nih.gov/RefSeq/ } \\
\text { Freely searchable, some content that } \\
\text { it links to requires additional access privileges. }\end{array}$ & $\begin{array}{l}\text { The American NIH database of DNA, RNA and protein } \\
\text { sequences, provides detailed information on many of } \\
\text { these genes and proteins. }\end{array}$ \\
\hline The Tumor Gene Family of Databases & $\begin{array}{l}\text { http://www.tumor-gene.org/Oral/oral.html } \\
\text { Freely searchable. }\end{array}$ & $\begin{array}{l}\text { A set of databases of tumor suppressors, potential } \\
\text { oncogenes and cancer-inducing mutations. }\end{array}$ \\
\hline
\end{tabular}

Expression levels of biomarkers analyzed in the datasets can be linked to clinically relevant parameters including sample site, clinical outcomes, molecular subtypes, pathological subtypes, drug sensitivities, and patient demographics.

\section{CDH11 is overexpressed in cancer tissue compared to} normal tissue of patients in HNSCC

A summary of representative histograms detailing expression levels in individual studies used to compile the metaanalyses of CDH11 is shown in Fig. 2A. Table 2 illustrates systematic overexpression of CDH11 in HNSCC, including a meta-analysis of the expression level in cancer vs. normal across multiple datasets.

\section{CDH11 is expressed in HNSCC tissue}

In silico data suggested that $\mathrm{CDH} 11$ is overexpressed in HNSCC. In order to validate the in silico findings, the expression of CDH11 was investigated in tissues. In initial studies, the antibody was optimized for immunohistochemistry on paraffin-embedded colon cancer tissue and associated tumor stroma, which are known to express CDH11 (19). After finding that CDH11 appropriately stained these positive control tissues, a TMA containing human HNSCC tissues and normal oral tissues was immunostained. CDH11 had low expression in normal tissue, and exhibited low, medium or high staining in HNSCC (Fig. 2B). HNSCC samples on the TMA exhibited a small but significant increase in intensity of staining, and proportion of positive HNSCC cells when compared to normal tissues (Fig. 2C). These data confirm that CDH11 is expressed in HNSCC tissues at higher levels than normal tissue.

Multiple HNSCC cell lines as well as a non-malignant immortalized keratinocyte (HOK16B) cell line were immunoblotted with the CDH11 antibody (Fig. 3A). These data showed that CDH11 was upregulated in $4 / 7$ HNSCC cell lines compared to HOK16B. Invasion is a significant 

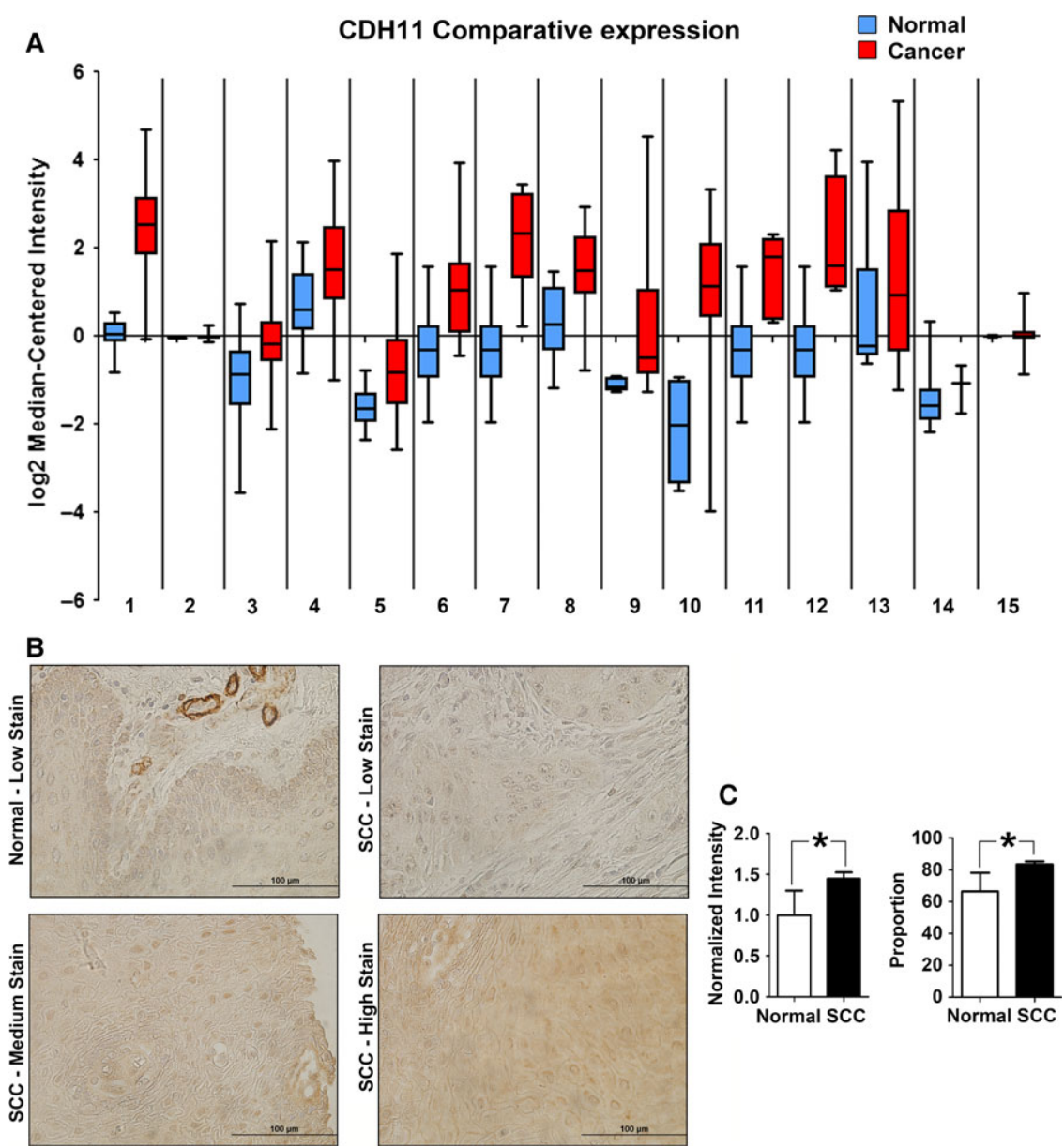

Figure $2 \mathrm{CDH} 11$ is expressed in cancer. (A) Meta-analysis showing CDH11 upregulation in cancer compared to normal tissue. Individual study sets and accession numbers are identified in Table 2. (B) A TMA with HNSCC and normal tissues was incubated with CDH11 antibody followed by DAB detection. $($ bar $=100 \mu \mathrm{m})(\mathrm{C})$ The intensity and proportion were scored $(* P<0.05)$.

phenotype in HNSCC and is correlated with EMT and loss of cell-cell adhesion. Since CDH11 differentially modulates invasion, migration and metastasis in multiple cancers $(4-7$, 20), we investigated the correlation between expression of EMT markers and CDH11. Epithelial marker E-cadherin and mesenchymal marker Twist-1, and actin as a loading control, were immunoblotted (Fig. 3B) in HNSCC lysates. The data show that the E-Cadherin is upregulated in UMSCC-(11A, 14A, 17B, 29, 47) cell lines and Twist-1 was downregulated in UM-SCC-(1,11A, 14A, 17B, 29) cell lines compared to HOK16B; 4/5 of these cell lines [UMSCC-(11A, 14A, 17B, 29)] showed an inverse relationship between Twist-1 and E-cadherin. To investigate the functional role of $\mathrm{CDH} 11$, we analyzed HNSCC datasets in Oncomine $^{\mathrm{TM}}$ for potential correlation between $\mathrm{CDH} 11$ and EMT markers, E-cadherin and Twist- 1 . We found a dramatic positive correlation between $\mathrm{CDH} 11$ and $\mathrm{E}$ cadherin (Fig. 3C) and a significant negative correlation between CDH11 and Twist-1 (Fig. 3D). The data suggested that the high level of CDH11 may promote E-cadherin and repress Twist-1 expression.

To confirm the bioinformatics data, UM-SCC-29 and UM-SCC-47, which express low and high CDH11 (Fig. 3A), were selected for functional studies. Two individual siRNAs were screened and downregulation was verified by immunoblot (Fig. 3E,F). After CDH11 was downregulated, epithelial and mesenchymal markers were examined to determine whether $\mathrm{CDH} 11$ negatively regulates EMT (Fig. 3G,H). In UM-SCC-29, which has low endogenous CDH11, Twist-1 is inversely correlated with CDH11 expression, but the direct correlation with E-cadherin and CDH11 is less prominent (Fig. 3G). In UM-SCC-47, which has high endogenous CDH11, E-cadherin is directly correlated with $\mathrm{CDH} 11$ and inversely correlated with Twist-1 expression (Fig. 3H). Importantly, a spindle-like EMT morphologic appearance was observed after knockdown of CDH11 in HNSCC cells (Fig. 4A). Furthermore, the positive correlation between $\mathrm{CDH} 11$ and E-cadherin was confirmed by immunofluorescence (Fig. 4B). Thus, CDH11 expression is correlated with a reverse EMT phenotype, including upregulated epithelial marker E-cadherin and downregulated mesenchymal marker Twist-1.

\section{CDH11 inhibits proliferation and invasion in vitro}

In order to investigate the functional role of CDH11, proliferation and invasion assays were performed in UMSCC-29 and UM-SCC-47 cell lines using two siRNAs, si7 and si8. Our data show that downregulation of CDH11 in 


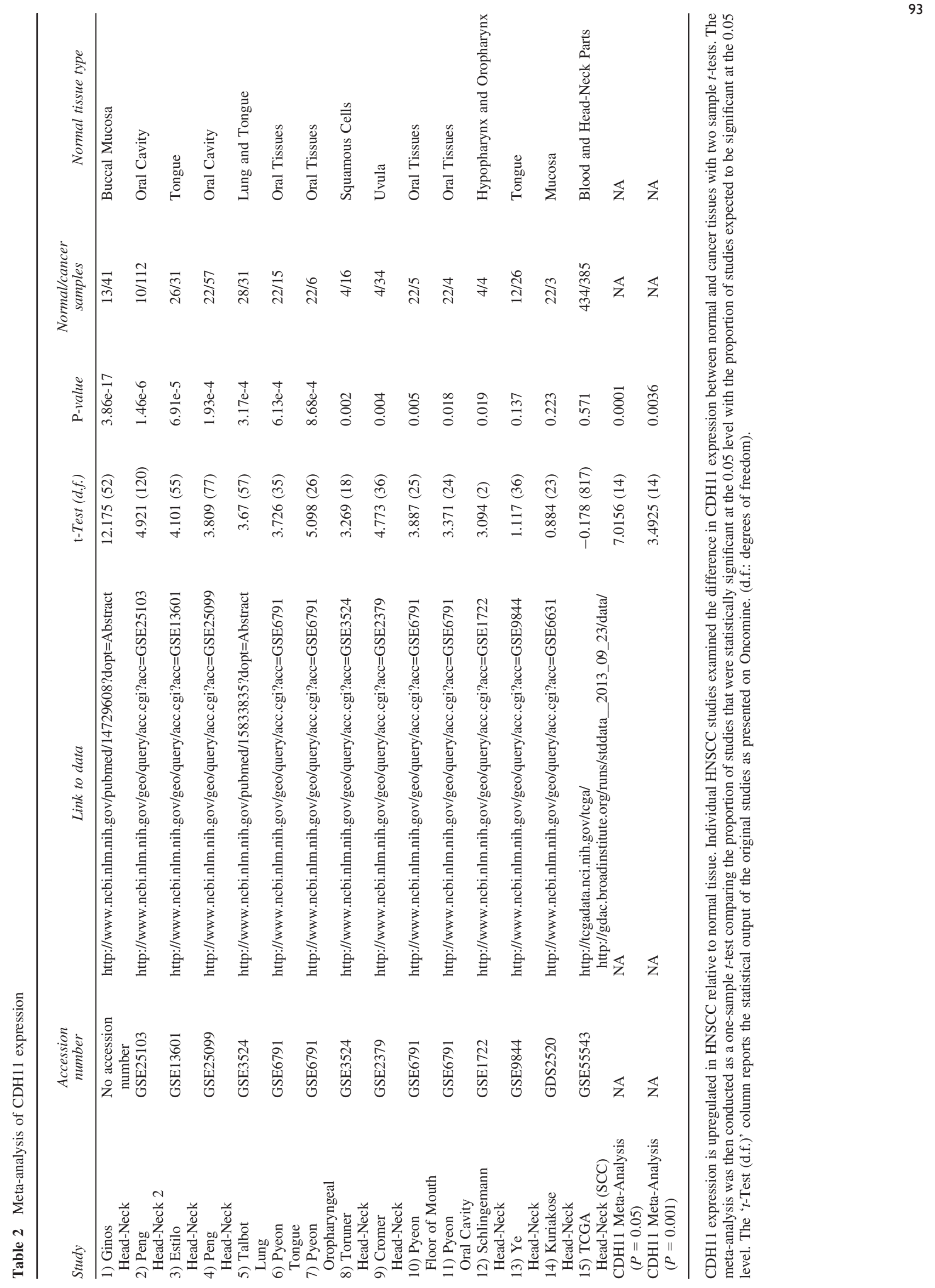


A

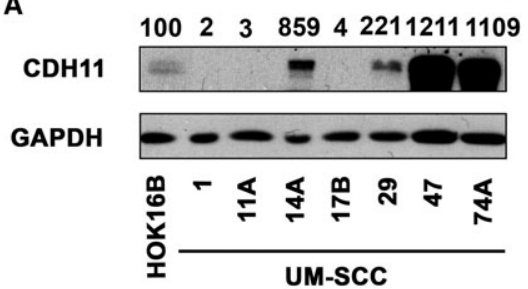

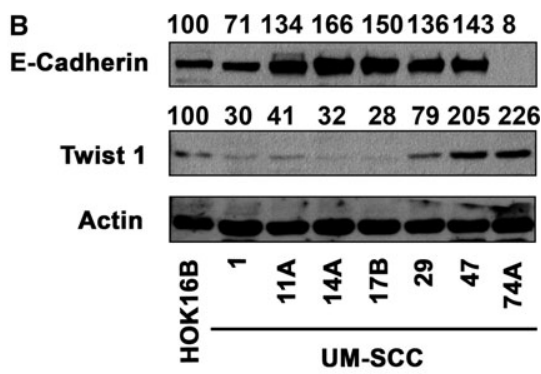

D

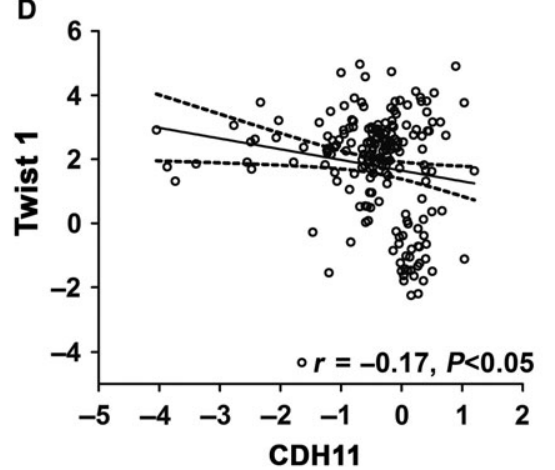

F

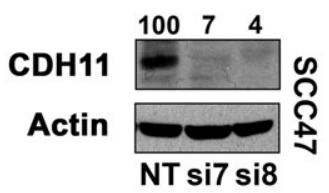

H

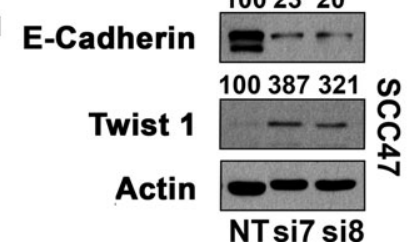

Figure 3 CDH11 expression correlates with a reverse epithelial to mesenchymal transition (EMT) phenotype. (A) Whole cell lysates of Immortalized keratinocytes (HOK16B) as well as UM-SCC-1, -11A, -14A, -17B, -29, -47, -74A were immunoblotted with anti-CDH11; GAPDH was used as a loading control. (B) Whole cell lysates of Immortalized keratinocytes (HOK16B) as well as UM-SCC-1, -11A, -14A, -17B, -29, -47, -74A were blotted with ECadherin, Twist-1, and actin as a loading control. (C, D) Spearman analysis for correlation between CDH11 and EMT markers (E-Cadherin and Twist-1) were conducted from multiple HNSCC database (C: Rickman Head-Neck, Ginos Head-Neck, Slebos Head-Neck, Pyeon Multi-cancer, Toruner Head-Neck, Ye Head-Neck, O`Donnell Oral, Schlingemann Head-Neck, $n=270$; D: Sengupta Head-Neck, Rickman Head-Neck, Pyeon Multi-cancer, Ye Head-Neck, Bittner Multi-cancer, $n=191$ ) from Oncomine (oncomine.org). Numbers represent $r$ values with significance $(P<0.0001, P<0.05$, respectively). Two individual siRNAs were investigated for efficiency in downregulating CDH11 in (E) UM-SCC-29 and (F) UM-SCC-47. After siRNA-mediated knockdown, cell lysates from UM-SCC-29 (G) and UM-SCC-47 (H) were immunoblotted with anti-E-Cadherin and anti-Twist-1. Actin was run as a loading control.

both cell lines by two different siRNAs significantly increased proliferation at 72 and $120 \mathrm{~h}$ (Fig. 4C). Invasion, an oncogenic phenotype that facilitates tumor spread, was also significantly enhanced by knockdown of CDH11 at $72 \mathrm{~h}$ (Fig. 4D). These findings are consistent with the reverse EMT phenotype observed in Fig. 3G,H. Together, the functional studies support a tumor suppressive role for CDH11 in HNSCC.

\section{Discussion}

Translational researchers are faced with the challenge of addressing the gap between experimental studies and implementation in clinical care. In silico investigations using multiple datasets provide researchers with a practical approach to enhancing benchtop investigations with clinically oriented data or to discovery of potential biomarkers. In the present study, we performed a meta-analysis of multiple datasets using the Oncomine ${ }^{\mathrm{TM}}$ platform. We observed that CDH11 is overexpressed in HNSCC relative to normal tissue across several datasets. Therefore, we investigated the expression and function of $\mathrm{CDH} 11$, and established that contrary to in silico findings, CDH11 has anti-proliferative and anti-invasive effects in HNSCC. In the context of a growing number of available genomic, transcriptomic, metabolomic and proteomic datasets, the present study highlights the importance of functionally validating in silico findings.

Cadherins are transmembrane glycoproteins that regulate homophilic intercellular adhesion by calcium-dependent 
A
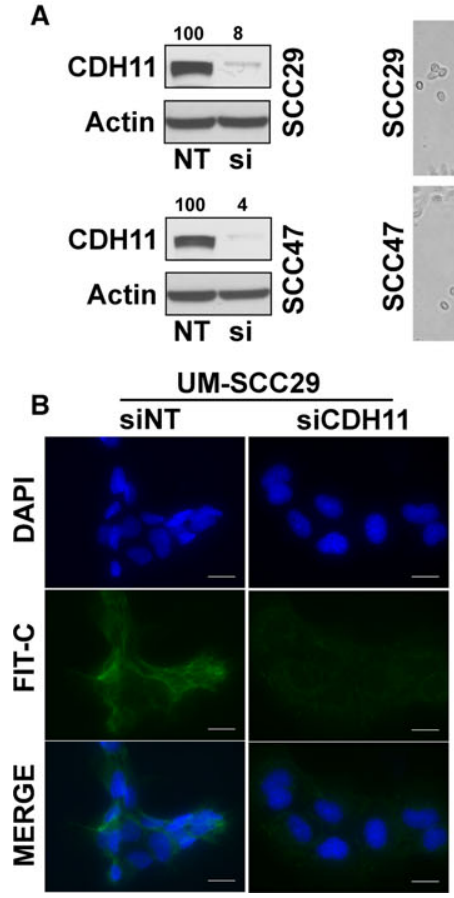

siNT
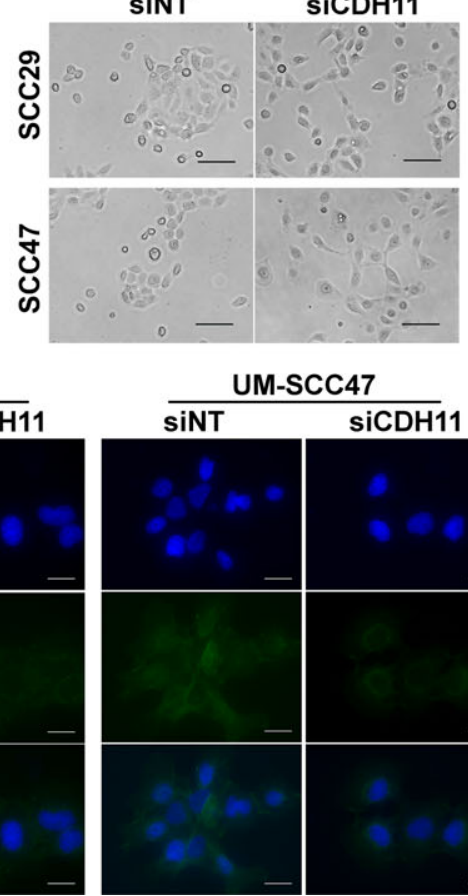

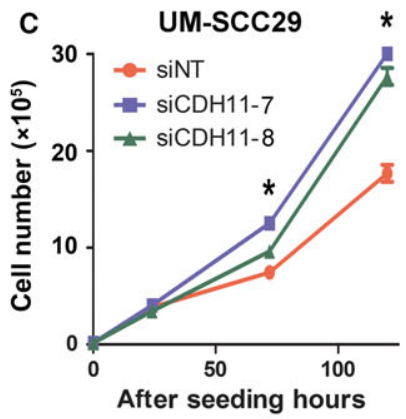

UM-SCC29

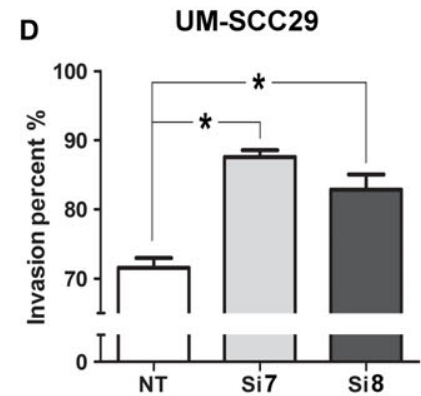

Figure 4 Downregulation of CDH11 in UM-SCC-29 and UM-SCC-47 cells promoted proliferation and invasion. (A) Morphology changes of UM-SCC-29 and UM-SCC-47 cells transfected with siNT or siCDH1 1 by phase-contrast microscopy. Original magnification, $\times 100($ bar $=200 \mu \mathrm{m})$. $(\mathrm{B}) \mathrm{UM}-\mathrm{SCC} 29$ and UM-SCC-47 cells were stained with Cadherin (green) antibodies and DAPI (blue) after transfection with siCDH11. (bar = 100 $\mu \mathrm{m})$. (C) UM-SCC-29 and UM-SCC-47 cells were transfected with siCDH11 (si7, si8) or NT siRNA. UM-SCC-29 and UM-SCC-47 cells were seeded in triplicate at 48 h after transfection in a 24-well plate and cells were counted at 1,3 , and 5 days after seeding. Total viable cells are shown. $* P<0.05$ at 72 h and at 120 h. (D) Cell invasion was assessed at $72 \mathrm{~h}$ in UM-SCC-29 and UM-SCC-47 cells transfected with siCDH11 (si7, si8) or NT siRNA. ${ }^{*}<0.05$ at $72 \mathrm{~h}$.

interactions (21). Cadherins contain a negatively charged domain, which binds $\mathrm{Ca}^{2+}(3)$. The cytoplasmic domain is divergent among cadherin family members (3). There are over 80 members in the cadherin superfamily (3). CDH11 is a member of the 'classical' cadherin subclass of the cadherin superfamily (21). Type I Classical cadherins include Ecadherin/cadherin 1, N-cadherin/cadherin 2, R-cadherin/ cadherin 4, P-cadherin or cadherin 3. Type II Classical cadherins include CDH11 (21). Other cadherins include protocadherins, desmosomal cadherins, seven-pass transmembrane cadherins, and Ret tyrosine kinase (21). Classical cadherins regulate cell-cell interactions, tissue homeostasis, and tissue morphogenesis; given these important roles, disruption of these proteins leads to disease (22). Cadherin homodimers on one cell bind homodimers on another cell to form transdimers that facilitate cell-cell adhesion (21).

The gene for CDH11 is located on chromosome 16 (3). CDH11 is usually expressed in mesenchymal cells and facilitates adhesion between these cells (23). CDH11 expression correlates with osteoblast-differentiation and promotes chondro-osteogenesis and inhibits adipogenesis (3, 21). Consistent with an oncogenic role, CDH11, promotes invasion of prostate cancer cells and metastasis to bone $(5,20)$. CDH11 expression is upregulated in colorectal tumors (24). In oral squamous cell carcinoma, a previous immunohistochemical study showed that CDH11 is overexpressed but did not perform functional studies (25). In the present study, in silico studies and TMAs of human HNSCC suggested that CDH11 is overexpressed in
HNSCC. Some HNSCC sections showed high CDH11 whereas in other sections, the expression was not as impressive. Together, these findings yielded an overall significant difference in expression of CDH11 between HNSCC and normal tissue. This is supported by immunoblot analysis showing overexpression of CDH11 in four out of seven HNSCC cell lines compared to normal keratinocytes. This suggests that overexpression of CDH11 is cancer-specific and is overexpressed in some and not in other HNSCCs. This is consistent with the heterogeneity of HNSCC and emphasizes the importance of personalized medicine in HNSCC. Importantly, in functional studies, we found that downregulation of CDH11 promotes proliferation and enhances invasion, consistent with a tumor suppressor role for CDH11. These findings are consistent with the recently reported loss of $\mathrm{CDH} 11$ expression in melanoma as a widespread event (26). Similarly, CDH11 undergoes genomic deletion in retinoblastomas (6) and its loss correlates with increased invasion. Studies in retinoblastoma (6) and malignant pheochromocytoma (27) showed that CDH11 potently suppresses tumor metastasis. Moreover, CDH11 expression reduced metastatic potential in lung cancer and represented a beneficial prognostic factor in osteosarcoma (28). Since the oncogenic vs. tumor suppressive function of CDH11 varies by cancer, it is important to consider the specific cancer type. Furthermore, different tumors within a subtype may differ in CDH11 expression. In the emerging area of personalized medicine, variation in CDH11 expression may be relevant to tumor behavior. 
Data mining of publically available datasets was used to nominate novel oncogenes having a role in epithelialmesenchymal transition in HNSCC. This study was the first to analyze the expression of $\mathrm{CDH} 11$ using an in silico experiment and verify its tumor suppressor role in HNSCC. In this role, $\mathrm{CDH} 11$ inhibits invasion and proliferation in HNSCC. Interestingly, CDH11 expression is inversely correlated with EMT, which has been shown to be an important event in invasion (29). This may occur via the Wnt/ $\beta$-catenin pathway. A previous study showed that $\mathrm{CDH} 11$ is a functional tumor suppressor and an important regulator of $\mathrm{Wnt} / \beta$-catenin signaling involved in EMT, with frequent epigenetic inactivation in common carcinomas (7).We showed previously that $\beta$-catenin promotes invasion in HNSCC (30). Future studies are needed to investigate this possibility.

In summary, we analyzed CDH11 expression in HNSCC by meta-analysis of fifteen datasets in the Oncomine ${ }^{\mathrm{TM}}$ database. These findings were verified by expression studies in multiple cell lines and HNSCC tissue. Importantly, we observed that CDH11 is a tumor suppressor in functional studies. Further investigations are needed to determine the mechanism of CDH11-mediated inhibition of invasion.

\section{Supporting Information}

Additional Supporting Information may be found in the online version of this article:

Table S1 The clinico-pathological parameters of the HNSCC cases and CDH11 intensity and proportion scores.

\section{References}

1. Cirillo N. Merging experimental data and in silico analysis: a systems-level approach to autoimmune disease and cancer. Expert Rev Clin Immunol 2012; 8: 361-72.

2. Rhodes DR, Kalyana-Sundaram S, Mahavisno V, et al. Mining for regulatory programs in the cancer transcriptome. Nat Genet 2005; 37: 579-83.

3. Yagi T, Takeichi M. Cadherin superfamily genes: functions, genomic organization, and neurologic diversity. Genes Dev 2000; 14: 1169-80.

4. Pishvaian MJ, Feltes CM, Thompson P, et al. Cadherin-11 is expressed in invasive breast cancer cell lines. Cancer Res 1999; 59: 947-52.

5. Huang CF, Lira C, Chu K, et al. Cadherin-11 increases migration and invasion of prostate cancer cells and enhances their interaction with osteoblasts. Cancer Res 2010; 70: 45809 .

6. Marchong MN, Yurkowski C, Ma C, et al. Cdh11 acts as a tumor suppressor in a murine retinoblastoma model by facilitating tumor cell death. PLoS Genet 2010; 6: e1000923.

7. Li L, Ying J, Li H, et al. The human cadherin 11 is a proapoptotic tumor suppressor modulating cell stemness through Wnt/beta-catenin signaling and silenced in common carcinomas. Oncogene 2012; 31: 3901-12.

8. Cromer A, Carles A, Millon R, et al. Identification of genes associated with tumorigenesis and metastatic potential of hypopharyngeal cancer by microarray analysis. Oncogene 2004; 23: 2484-98.

9. Estilo $\mathrm{CL}$, Oc P, Talbot $\mathrm{S}$, et al. Oral tongue cancer gene expression profiling: identification of novel potential prognosticators by oligonucleotide microarray analysis. BMC Cancer 2009; 9: 11.

10. Ginos MA, Page GP, Michalowicz BS, et al. Identification of a gene expression signature associated with recurrent disease in squamous cell carcinoma of the head and neck. Cancer Res 2004; 64: 55-63.

11. Kuriakose MA, Chen WT, He ZM, et al. Selection and validation of differentially expressed genes in head and neck cancer. Cell Mol Life Sci 2004; 61: 1372-83.

12. Peng $\mathrm{CH}$, Liao CT, Peng SC, et al. A novel molecular signature identified by systems genetics approach predicts prognosis in oral squamous cell carcinoma. PLoS One 2011; 6: e23452.

13. Pyeon D, Newton MA, Lambert PF, et al. Fundamental differences in cell cycle deregulation in human papillomavirus-positive and human papillomavirus-negative head/ neck and cervical cancers. Cancer Res 2007; 67: 4605-19.

14. Schlingemann J, Habtemichael N, Ittrich C, et al. Patientbased cross-platform comparison of oligonucleotide microarray expression profiles. Lab Invest 2005; 85: 1024-39.

15. Talbot SG, Estilo C, Maghami E, et al. Gene expression profiling allows distinction between primary and metastatic squamous cell carcinomas in the lung. Cancer Res 2005; 65: 3063-71.

16. Toruner GA, Ulger C, Alkan M, et al. Association between gene expression profile and tumor invasion in oral squamous cell carcinoma. Cancer Genet Cytogenet 2004; 154: 27-35.

17. Ye H, Yu T, Temam S, et al. Transcriptomic dissection of tongue squamous cell carcinoma. BMC Genom 2008; 9: 69.

18. Banerjee R, Russo N, Liu M, et al. TRIP13 promotes error-prone nonhomologous end joining and induces chemoresistance in head and neck cancer. Nat Commun 2014; 5: 4527.

19. Torres S, Bartolome RA, Mendes M, et al. Proteome profiling of cancer-associated fibroblasts identifies novel proinflammatory signatures and prognostic markers for colorectal cancer. Clin Cancer Res 2013; 19: 6006-19.

20. Chu K, Cheng CJ, Ye X, et al. Cadherin-11 promotes the metastasis of prostate cancer cells to bone. Mol Cancer Res 2008; 6: 1259-67.

21. Marie PJ, Hay E, Modrowski D, et al. Cadherin-mediated cellcell adhesion and signaling in the skeleton. Calcif Tissue Int 2014; 94: 46-54.

22. Yap AS, Gomez GA, Parton RG. Adherens junctions revisualized: organizing cadherins as nanoassemblies. Dev Cell 2015; 35: 12-20.

23. Alimperti S, Andreadis ST. $\mathrm{CDH} 2$ and $\mathrm{CDH} 11$ act as regulators of stem cell fate decisions. Stem Cell Res 2015; 14: $270-82$.

24. Bujko M, Kober P, Mikula M, et al. Expression changes of cell-cell adhesion-related genes in colorectal tumors. Oncol Lett 2015; 9: 2463-70.

25. Choi P, Jordan CD, Mendez E, et al. Examination of oral cancer biomarkers by tissue microarray analysis. Arch Otolaryngol Head Neck Surg 2008; 134: 539-46.

26. Mueller DW, Bosserhoff AK. MicroRNA miR-196a controls melanoma-associated genes by regulating HOX-C8 expression. Int J Cancer 2011; 129: 1064-74.

27. Sandgren J, Andersson R, Rada-Iglesias A, et al. Integrative epigenomic and genomic analysis of malignant pheochromocytoma. Exp Mol Med 2010; 42: 484-502. 
28. Nakajima G, Patino-Garcia A, Bruheim S, et al. CDH11 expression is associated with survival in patients with osteosarcoma. Cancer Genomics Proteomics 2008; 5: 37-42.

29. de Craene B, Berx G. Regulatory networks defining EMT during cancer initiation and progression. Nat Rev Cancer 2013; 13: 97-110.

30. Goto M, Mitra RS, Liu M, et al. Rap1 stabilizes beta-catenin and enhances beta-catenin-dependent transcription and invasion in squamous cell carcinoma of the head and neck. Clin Cancer Res 2010; 16: 65-76.

\section{Acknowledgements}

We thank Amirtha Hariharan for technical help. This work was supported by NIDCR DE019513, DE018512-01, DE022567 (NJD), DE021293 (CSS) and the Elizabeth Caroline Crosby Fund.

\section{Conflict of interest statement}

The authors declare that there are no conflict of interests. 